\title{
Systems Engineering on the Dark Side of the Moon
}

\author{
Donna H. Rhodes \\ International Council on Systems Engineering
}

\begin{abstract}
Systems engineering is an essential discipline for both defense and commercial businesses. To date, it has not been possible for INCOSE to establish as strong a leadership presence in the commercial world as it has in the defense world. This is clearly related to the fact that heritage membership of INCOSE came largely from a defense orientation. It is also related to the author's premise that just as our observer point on earth limits our ability to see the dark side of the moon, our point of observation in our professional domain limits the capacity to truly see the 'other side'. The author, having a seventeen year career in the defense industry, recently transitioned to a position in commercial software product development. The observations and insights gained are the subject of this paper, and advocate further comparative research and study of systems engineering as a means to enrich the overall theory and practice.
\end{abstract}

\section{THE OBSERVER'S DILEMMA}

Why can't we see the dark side of the moon? The reason is that the observer's viewpoint on earth restricts this. The moon travels around the earth once every 29 days. The moon, attracted by the Earth's gravitational pull, travels with the earth and circles the sun every 365 days. The moon rotates completely on its axis only once during each trip around the earth. When we look at the moon, we always see the same side. This is because the moon turns once on its axis in the same time that it circles the earth. Unlike the earth, which rotates 365 times during each rotation around the sun, the moon only rotates once. This results in your observation...only one side of the moon can ever be seen from a specific point on earth.

Similarly, I assert that when one is "standing in the middle of the defense business" it is just not possible to fully understand the Commercial Product Development (CPD) world. As such, it has been difficult for INCOSE as an organization having a heritage strongly rooted in the defense industry to make connections and contributions oriented toward the world of CPD. As a first step, comparative study of systems engineering in these two worlds is essential.

This is particularly true in regard to $\mathrm{CPD}$, where organizations develop "smaller" products designed to satisfy a particular business customer or consumer need, as contrasted to large Commercial Systems
Integration (CSI) organizations developing systems such as commercial aircraft or automobiles. We understand that CSI requires a systems approach. (Clausing, Axelband, and Campbell 1999) describe the CPD organization as "a company that faces sufficient strong global competitors and is little constrained by external rigidities." CPD is at the most distant end of the spectrum from large defense systems, yet here too, there are increasing challenges of a systems nature.

I think there is great value in discovering that which connects us as systems professionals across processes, practices, and technologies - and across boundaries of geography, domain, profession, and more. Looking at systems engineering from diverse domain perspectives helps us understand not only the points of separation, but also the points of convergence. Thoughts in this presentation are based on two years of personal experience in commercial software product development. Further, these experiences relate more to CPD rather than the challenges found in CSI, where the commercial practices tend to be more aligned with defense practices. Rather than being "absolutes" I hope these thoughts will lead to additional dialogue and inquiry.

\section{UNIQUE CHALLENGES IN CPD}

There are many unique challenges in CPD. Optimizing the customer's needs and interests with the off-the-shelf product is one such challenge. Commercial businesses can not afford to build 'one-offs' for each customer, and therefore requirements engineering strategies must find the way to optimize satisfying the largest number of potential customers with the minimum number of product features. Achieving this is an intellectual juggling act that requires the CPD organization to continuously evaluate incoming information as new potential customers are identified and new technologies and competitors come into the marketplace. CPD must accommodate the rapidly changing customer base where the profile of customer may rapidly change.

Increasingly commercial product developers must offer a "total solution" to a customer business problem (or individual consumer need) where previously they simply sold one or more products, thus introducing new systems integration issues. Where in the past, many companies sold everything as single products, today these same companies must learn to effectively bundle 
multiple products and associated services into "offers", often with variations targeted at specific market segments. The challenges of designing and testing for interoperability are rapidly growing in commercial companies.

Maximizing the global collaboration of highly diverse teams on several levels is critical, as is creating designs/products that tolerate variability in multiple dimensions. Competition is changing in the "legacy INCOSE world", but in CPD there is even a greater challenge in responding to rapidly morphing competition - alliances, mergers, and partnerships change on a very frequent basis.

\section{TECHNICAL APPROACHES}

The technical approaches used by defense organizations and CPD are similar in that both transform a need into a set of requirements. In turn, these requirements drive the development of a design leading to implementation of a final product/system that will satisfy an end-user.

CPD has a strength in analyzing the gaps between the commercial product offering and needs of a customer who will be acquiring the product. This includes understanding variations based on market segmentation of the customer base. Requirements engineering begins with the set of initial market needs. Desired capabilities are then detailed, and features that will provide such capability are then elaborated. Once features are defined, the functional and non-functional requirements are detailed. The capability level view is critical in ensuring that the resulting product will serve the market.

Engineering approaches in CPD are oriented around an end goal of supporting the majority of customer's needs with an off-the-shelf solution. As pointed out by (Chew 1997), large commercial companies will have formal and very aggressive requirements benchmarking programs, with product clinics and focus groups. Use cases are frequently employed to focus on how the end-users interact with the system. Early development of prototypes and controlled introductions of new products are used effectively as strategies to help CPD to realize a product that will be well-accepted in the marketplace. As noted by (Honour 1995), "customers do not directly guide the development, but they are the final arbiters of project success through their decisions to buy or not to buy."

Defense engineering techniques have strong emphasis in decomposition. Interface requirements and design is a well-established part of the overall requirements and design activities. Traceability and the use of verification cross-reference matrices are a basic part of the requirements engineering process used by defense. Approaches are employed to drive the set of requirements toward providing maximum functionality for an individual customer's investment. Formal techniques, such as AHP, are used in requirements prioritization and technical trade-offs.

Previous studies on comparison of software acquisition methods in commercial versus defense such as (Ferguson, J., DeRiso, M, 1994) have noted that in best commercial practice, specifications are modified by the knowledge of availability of existing products, where in defense industry there is little to no regard for existing products. The authors note that in commercial, there is "more willingness to adjust requirements based on availability of products and thus to field a system, sooner and evolve it to include more capability".

Table 1 gives some examples of the strengths each domain of practice may contribute to the other in the area of technical approach.

\begin{tabular}{|l|l|}
\hline $\begin{array}{l}\text { Defense can look to } \\
\text { CPD to improve... }\end{array}$ & $\begin{array}{l}\text { CPD can look to } \\
\text { Defense to improve... }\end{array}$ \\
\hline Defining capability level & Defining interface \\
information & information \\
\hline Understanding end-user & $\begin{array}{l}\text { Decomposition and } \\
\text { relationships between } \\
\text { interaction with system } \\
\text { through use cases }\end{array}$ \\
\hline Validation via prototypes & Verification/validation \\
and product demos & planning methods \\
\hline Use of market & Use of formal methods \\
intelligence data in & for aiding decision \\
decision analysis & analysis \\
\hline
\end{tabular}

\section{Table 1: Technical Approaches: Examples where Defense and CPD can learn from each other.}

\section{MANAGEMENT APPROACHES}

Strategies used in CPD are intensely market-driven, with strong approaches for technology roadmapping. These roadmaps describe the evolution of a product in successive releases, and are continuously evolving to accommodate new business opportunities. Decisions are made rapidly with best available market data as the basis. The criteria for decisions tend to be much less rigorous than those used in a defense organization, and decisions are often changed as more information becomes available. The tendency and the risk in CPD is that decisions are made too rapidly, and without truly adequate information. Yet, the nature of the business demands this, so rapid alternative plans and mitigation techniques must always be in the back pocket.

In this rather volatile environment, CPD uses stage-gates to introduce some measure of control. Stage-gates are formal decision checkpoints in a product lifecycle, where business case and technical information are used to discern whether a project 
should continue to next phase. The most successful CPD processes encourage a large pipeline of ideas entering the business, with several decision gates in early phases to narrow down the candidate projects into the most technically and financially viable ones. In less mature CPD organizations, stage-gates may be in place, but lack well-specified decision criteria. In mature CPD companies, the criteria address a robust and wellintegrated set of technical and business factors.

The defense business is very much driven by specific opportunities, and these opportunities tend to occur in as families of linked projects. Information on spending profiles of the acquiring organizations for these future opportunities is the market knowledge base for defense. Large investments will be made in anticipation of future funding in a given area. The emergence of opportunities spans a much greater timeframe in defense. The investment required is both significant and potentially more at-risk than in CPD. Thus, planning is slower paced, and much more rigorous in nature. Decisions are often deferred or delayed due to insufficient information, and there is a risk of planning being in a state of paralysis.

(Ferguson and DeRiso 1994) point out a key difference in commercial and defense acquisition. Commercial is looking to produce a product through whatever means possible to achieve profit and overall business goals, whereas defense must ensure "fairness" in the process and the maintenance of public trust. This certainly introduces several constraints in how such projects must be managed.

Project management and planning are very dynamic activities in CPD. The strength lies in the versatility and openness to new ideas, just-in-time information, and leveraging emerging trends in the marketplace. The risk lies in lack for formality and planning using unvalidated or inadequate information. As such, it is difficult to measure progress against plan and build historical baselines required to improve future planning efforts.

Planning in defense organizations is more formalized and "scientific" in nature. Formal techniques are more prevalent, and the use of planning and management tools is more pervasive. Variances against plan are intensely examined, and metrics related to planning (CPI, SPI, on-time delivery, etc.) are integral to the overall management approaches, while in CPD these are likely to have been introduced more recently, and tend to be found only in more mature organization.

Defense organizations have higher motivation to develop formal plans, conduct formal reviews, and report progress metrics because these are likely to be requirements of doing business with a given customer who is funding the entire project. The customer will want to be the approver of plans and an involved participation in reviews. Monthly metrics reporting is likely to be required by the savvy defense-contracting agency.

In CPD, the customer comes into the picture as a key reviewer at a much later stage. The planning and implementation have likely progressed to a point where the product is ready for a controlled introduction. The customer is interested in the quality metrics and delivery completion schedule. It is up to the internal CPD management team to monitor the project development and launch plans, and as such, there tends to be less of a sense of urgency and formality around the planning and progress reporting. Any urgency in CPD is typically driven by competitive time-to-market pressure, and exhibits primarily as demands on the performance of the development team to get the product out the door. And it should be emphasized that pure time to market is not as important as what (Honour 1995) described as "intelligent selection of right timeto-market". The set of metrics and algorithms for determining the right time is, I believe, an interesting area for further research.

Table 2 gives some examples of the strengths each domain of practice may contribute to the other in the area of management approach.

\begin{tabular}{|l|l|}
\hline $\begin{array}{l}\text { Defense can look to } \\
\text { CPD to improve... }\end{array}$ & $\begin{array}{l}\text { CPD can look to } \\
\text { Defense to improve... }\end{array}$ \\
\hline $\begin{array}{l}\text { Using formal technology } \\
\text { roadmapping techniques }\end{array}$ & $\begin{array}{l}\text { Analysis of opportunity } \\
\text { dependencies/interaction }\end{array}$ \\
\hline $\begin{array}{l}\text { Stage-gates as integrated } \\
\text { technical/business } \\
\text { checkpoints }\end{array}$ & $\begin{array}{l}\text { Specificity of the criteria } \\
\text { used at formal milestone } \\
\text { reviews }\end{array}$ \\
\hline $\begin{array}{l}\text { Balancing the use of } \\
\text { qualitative/experiential } \\
\text { data with formal data }\end{array}$ & $\begin{array}{l}\text { Assessing progress } \\
\text { against plan with SPI, } \\
\text { CPI, and other measures }\end{array}$ \\
\hline
\end{tabular}

Table 2: Management Approaches: Examples where Defense and CPD can learn from each other.

\section{INFRASTRUCTURE}

The engineering of systems requires strong technical and management approaches, as well as the infrastructure to support these. Infrastructure involves people, processes, tools, and metrics.

Defense organizations tend to have clearly defined roles and responsibilities, with well-mapped career paths for technical, management, and administrative professionals. Skill acquisition and training programs tend to be oriented around the particular role in a specific discipline. In CPD, roles and responsibilities are much more loosely defined, and individuals often move in and out of various roles as projects come and 
go, and phases of projects progress. Skills and training are centered around market-segment orientation, and on specific technology.

Processes in CPD tend to be less well defined than in defense, with the exception of the larger and mature commercial companies. Interestingly, some of the more process-mature commercial companies I have observed, are those that have linkage to defenseoriented businesses which have perhaps shaped the approaches of the commercial part of the organization. Defense organizations have stronger processes that tend to be linked to a specific discipline. Standards often have a process orientation, and use of capability models is fairly widespread. In CPD, standards tend to be quality oriented or technology oriented, rather than process oriented. Use of capability models is much more limited in CPD, although these can be of great benefit if appropriately tailored.

The use of tools in the engineering process is more prevalent in the defense organization than it is in the smaller CPD company. Size of projects and level of investment are two of the factors behind this. Requirements tools, software and hardware design tools, testing tools, metrics tools, planning tools, problem trackers, action trackers, financial tracking systems, document management tools, configuration management tools, and many others are in wide use in most defense contractor companies. In CPD, the tools in use tend to be fewer and less sophisticated. Requirements tools are beginning to be used more often than in the past, but may be homegrown tools or inadequately/improperly used commercial tools. Automated testing and code generation tools are of high interest in commercial, and there is a greater potential for effective use of these where non-mission critical software and hardware is being developed. In CPD, there is better use of collaboration and communication tools to support teams that are spread across geographies and timezones.

The defense business has a longer history of process-based metrics programs. Many organizations have built good historical baselines of metrics data as a basis for planning and estimation. Predictive models employ this data to yield better plans for new projects. Defense organizations are driven to build, sustain, and grow metrics programs by the process standards and maturity models, which are levied by the customer. While lagging in metrics from a timeline perspective, the commercial companies are now putting heavy emphasis on metrics. In telecommunications, for example, the TL9000 Quality Standard includes an extensive set of metrics, which must be collected and reported. The "Balanced Scorecard" is another metrics approach that is beginning to be widely used in commercial. Historical baselines can be built more rapidly in CPD, as product lifecycles are fairly short.
As such, it can be concluded that while CPD is joining the metrics game much later than defense, we can expect CPD to soon surpass defense companies in the robustness of metrics programs and possibly the extensiveness of historical baselines as well.

Table 3 gives some examples of the strengths each domain of practice may contribute to the other in the area of infrastructure.

\begin{tabular}{|l|l|}
\hline $\begin{array}{l}\text { Defense can look to } \\
\text { CPD to improve... }\end{array}$ & $\begin{array}{l}\text { CPD can look to } \\
\text { Defense to improve... }\end{array}$ \\
\hline $\begin{array}{l}\text { Expanding engineering } \\
\text { roles more into the } \\
\text { marketing realm }\end{array}$ & $\begin{array}{l}\text { Definition of roles, } \\
\text { responsibilities, and } \\
\text { career paths }\end{array}$ \\
\hline $\begin{array}{l}\text { Defining processes in } \\
\text { less prescriptive manner }\end{array}$ & $\begin{array}{l}\text { Using process standards } \\
\text { to improve practices }\end{array}$ \\
\hline $\begin{array}{l}\text { Robustness of metrics } \\
\text { program using Balanced }\end{array}$ & $\begin{array}{l}\text { Using historical metric } \\
\text { data to build predictive } \\
\text { Scorecard approach }\end{array}$ \\
\hline $\begin{array}{l}\text { Using tools to enhance } \\
\text { collaboration between } \\
\text { distributed teams }\end{array}$ & $\begin{array}{l}\text { Using engineering and } \\
\text { management tools more } \\
\text { effectively in practice }\end{array}$ \\
\hline
\end{tabular}

Table 3: Infrastructure Approaches: Examples where Defense and CPD can learn from each other.

\section{SHARED CHALLENGES}

There are a number of key differences in commercial product development practices when compared to defense practices, but there are similarities and shared challenges, as well. The defense organizations and CPD organizations are both undergoing change driven by technology, globalization, and changing dynamics of the world in this new century. The ability to rapidly adopt and integrate new technology is increasingly important for both defense and CPD. Understanding the customer's requirements is the prerequisite for success in either world. Processes, best practices, knowledge management, and workforce skill development are key discriminators. And, in both worlds, most problems - technical and organizational are rooted in managing interfaces and dealing with change. Scenario development, architecture development, requirements management, risk management, trade studies, and many other fundamental practices are equally applicable in defense and commercial. However, we must keep in mind as pointed out by (Lentz 2000), that these may not all be applied in the same order or with the same focus. (Parth 1998) states a similar thought, "many of the approaches used in defense can be adapted and tailored to commercial development when it makes sense to do so...the differences lie not so much in what is done as 
in how it is done." Understanding the nuances of these practices and approaches in context of domain of practice will serve to further the body of knowledge as a whole.

\section{IMPLICATIONS FOR INCOSE}

In thinking about the similarities and differences, I believe there are a number of implications for INCOSE. "Design for change" and "evolutionary development" approaches and methods are important areas of research, as are collaboration methods and tools for global organizations. Although much has already been written on the subject, we have a continued need to expand our body of knowledge on requirements elicitation techniques -- both formal and "soft systems". Convergence techniques for eliminating the gap between acquirer's need and provider's solution is another key area of interest to both commercial and defense. Customization of maturity models and assessment methods to meet unique organizational needs is a valuable area of consideration. And, critically important for every organization, is the means to develop systems leaders for tomorrow. I believe INCOSE is working in several of these areas, and we need to expand these efforts, as well as think about them in very broad application context. As stated by (Rochecouste 1999), "INCOSE cannot become totally international without successfully addressing the systems engineering imperatives of the commercial world."

It strikes me that most studies done to date seem focused on how commercial best practices can be applied in the defense business. For example, Clausing, et al 1999) strongly state the case for how CPD methods can improve defense/aerospace systems engineering. Some of the more rigorous research to date has been focused on software practices, although most findings can be generalized to the engineering of systems. An example is the Product Line Practices of the Software Engineering Institute recently published in (Clements and Northrop, 2001).

Clearly commercial practices are influencing defense practices but there is also significant value to be gained for commercial (both in CPD and CSI) to adopt/adapt practices from defense. This belief is supported by (Parth 1998), who captures what I view as the pleasurable aspect of applying practices from the defense world in commercial: "we have the freedom to pick and choose which approaches, methods, and techniques will give us the information we need to develop products and grow the business, but not be driven by rules and regulations that do not apply."

Quite understandably, studies performed by governments or companies are looking for practices to flow in one direction. INCOSE has made significant progress in the past several years in characterizing systems engineering across numerous commercial domains, as evidenced by the INCOSE Systems Engineering Applications Profiles (INCOSE SE Applications Working Group, 2000) and the significant number of papers and panels in symposia. I think INCOSE is in an excellent position to lead further inquiry of a comparative nature, and to take a balanced perspective in comparing systems engineering practices and techniques to find mutually beneficial ideas and convergence approaches. Academia can also bring this unbiased view, and there is a need for further comparative studies and research in methodologies with broad applicability to both defense and commercial industries.

We also face a problem that is not unlike what (C.P. Snow 1959) expressed as a concern that the two cultures (of "literacy intellectuals" and "scientists") had no common ground for communication. In a sense, CPD and Defense sometimes behave like two different cultures. Continued dialogue should be fostered; one example was the Panel Session held at the INCOSE 1999 symposium on Systems Engineering in the Public and Private Sector. Another excellent event was the May 2000 Conference on "Systems Approach to Product Innovation and Development in HyperCompetitive Environments" sponsored by INCOSE Colorado Front Range Chapter. Language remains a barrier, but we must develop an ability to converse with each other, accepting that neither language is the 'correct' one.

Contributions INCOSE can make to further the vision of a "global" systems engineering include:

1. Lead or sponsor comparative studies of systems engineering methodologies and techniques, toward the goal of establishing a robust discipline.

2. Promote the value of engineering education and professional experiences that provide practical experience with multiple domains of practice.

3. Continue to encourage both participation and leadership involvement of commercial organizations in INCOSE.

4. Seek opportunities for collaboration and alliances with commercially oriented professional socieities such as Project Management Institute (PMI) and Product Development \& Management Association (PDMA).

5. Assists in breaking down perceived barriers that exist through continued dialogue and interaction in initiatives that cross the domains of practice.

6. Provide our membership with access to knowledge based on multiple perspectives and the opportunities to network with practitioners from a wide variety of backgrounds.

7. Foster an open-minded view of the systems profession as a basic tenet of INCOSE, and embrace the approaches, practices, and languages 
of all "cultures" that practice systems engineering.

\section{SUMMARY}

It's true -our observer's point of view limits the ability to completely understand what lies on the "dark side of the moon". However, it is critically important that we work toward a more holistic understanding of the discipline and practice of systems engineering that is independent of the domain of practice. Most organizations can not survive in the 21st century without using a systems approach, and so systems engineering is a discipline that both commercial and defense must embrace. We need to focus on the practices, methods, and techniques that are applicable to both worlds. It is equally important to understand the uniqueness and differences, as it is to understand shared challenges and similarities. Based on my experiences, I believe each type of organization has much it can learn from the other. As systems practitioners and researchers, we must continue to enhance our understanding of systems engineering though examination of both convergent and divergent points of view. Further research and study of systems engineering in a comparative manner is needed to enrich the overall theory and practice in each domain.

\section{REFERENCES}

Chew, James, "Commercial Best Practices and the DoD Acquisition Process", Acquisition Review Quarterly, 1997

Clausing, D., Axelband, E., and Campbell, R.B., "Advances in Commercial Product Development Lessons for INCOSE Systems Engineering", INCOSE INSIGHT, Volume 2, Issue 3, Fall 1999

Clements, P. and Northrop, L., Software Product Lines, SEI Series in Software, 2001

Ferguson, J., and DeRiso, M., "Software Acquisition: A Comparison of DoD and Commercial Practices", Software Engineering Institute, CMU/SEI-94-SR-9

Honour, E., "Principles of Commercial Systems Engineering" Proceedings of the NCOSE Symposium, July 1995

INCOSE SE Applications Working Group, Systems Engineering Applications Profiles Version 3.0, International Council on Systems Engineering, July 2000

INCOSE Panel Session, Systems Engineering in the Public and Private Sector, Report of Panel at 1999 Symposium of International Council on Systems Engineering, E.Axelband, Moderator, 1999 http://www.incose.org.uk/incose99/panels.htm

Lentz, V., "Five Realities for Systems Engineering in Commercial Enterprises", Proceedings of the
INCOSE Symposium, July 2000

Parth, F., "Systems Engineering Drivers in Defense and in Commercial Practice", Systems Engineering, Volume 1, Number 1, 1998

Rochecouste, H., "Successful Development of Commercial Infrastructure Projects with Systems Engineering", Systems Engineering, Volume 2, Number 4, 1999

Snow, C.P., The Two Cultures, Cambridge University Press, 1959

\section{BIOGRAPHY}

Dr. Donna H. Rhodes is presently Director of Process Engineering at CSG Systems (formerly Kenan Systems/Lucent Technologies) in Cambridge, MA, where she is focused on addressing the increasing systems challenges in commercial product development. Previously she was the Systems Engineering Process Owner at Lockheed Martin Federal Systems, and managed the engineering centerof-competence, providing consultation to programs on engineering practices, technology, and metrics. Dr. Rhodes served as INCOSE President in 2000, and previously chaired the INCOSE Technical Board. She is presently INCOSE Director by Appointment for Strategic Planning. She is an Associate Editor of INCOSE's journal, Systems Engineering. She has published over 30 technical papers in the area of Systems Engineering, co-authored industry and company standards and guidebooks, and has been an invited speaker and panelist for numerous industry, government, university, and corporate activities. Dr. Rhodes received her M.S. and Ph.D. in Systems Science/Advanced Technology. She is a member of several university/industry advisory boards focused on establishing systems engineering graduate degree programs. 
